Medicine Updates

Faculty of medicine

July 2020,volume 2 , issue 2 https://muj.journals.ekb.eg

dean@med.psu.edu.eg

vice_dean_postgraduate@med.psu.edu.eg

DOI:10.21608/muj.2020.28821.1009

ISSN : 2682-2741

Submitted: $27 / 4 / 2020$

Accepted : 7/5/2020

Pages:1-14

\title{
Assessment of left ventricular mechanics and energetics in various types of left bundle branch block using echocardiography
}

Dr. Mahmoud Shehata Abd El Hamid Shehata lecturer of Cardiology and Angiology، Faculty of Medicine، University of Port Said

Prof. Dr. Mustapha Mohamed Nawar Professor of Cardiology and Angiology, Faculty of Medicine, University of Alexandria.

Prof. Dr. Luigi P. Badano Professor of cardiac, Thoracic and vascular science Faculty of Medicine, University ofPadova, Italy.

Dr. Eman Mohamed El-Sharkawy Assistant Professor of Cardiology and angiology Faculty of Medicine, University of Alexandria

Dr. Aly Ahmed Abo El Hoda Lecturer in Cardiology and Angiology, Faculty of Medicine University of Alexandria.,

\begin{abstract}
BACKGROUND and AIM: Recent data suggested that one third of patients meeting conventional electrocardiographic (ECG) criteria for left bundle branch block (LBBB) may be misdiagnosed and new, stricter, ECG criteria for LBBB have been proposed (Strauss's criteria). Accordingly, we used two-dimensional speckle tracking echocardiography (2DSTE), to compare left ventricular (LV) mechanics in patients with LBBB according to conventional versus Strauss's ECG criteria for LBBB.
\end{abstract}


METHODS: We studied 49 asymptomatic patients with incidental LBBB: 16 with conventional (mean age 69 years, 56\% were men) and 33 with Strauss's criteria (mean age 66 years, $61 \%$ were men), LV ejection fraction (LVEF) $\geq 45 \%$, and no significant obstructive coronary artery disease. Three-dimensional echocardiography was performed to measure LV end diastolic (LVEDV) and end systolic (LVESV) volumes, sphericity index (SpI), LV remodelling index (LVRI) and LVEF. 2DSTE was used to measure parameters of LV dyssynchrony: septal to posterior wall time to peak radial strain (SW-PW delay), longitudinal strain delay index (L-SDI), longitudinal strain rate dispersion index (L-SRDI) and LV mechanical dispersion (LVMD). In addition, we also measured LV cardiac work indices: global work efficiency (GWE), global work index (GWI), global constructive work (GCW), and global wasted work (GWW).

RESULTS: Among dyssynchrony parameters; L-SDI was significantly higher in patients with Strauss's criteria than in conventional one $(37 \pm 17 \%$ vs $25 \pm 10 \%$, respectively, $\mathrm{p}=0.007)$. Moreover, GWW tended to be higher in patients with Strauss's criteria than in conventional one $(325 \pm 132 \%$ vs $267 \pm 124 \%$ respectively, $p=0.079)$. In all patients, LVESV index was positively correlated with GWW $(\mathrm{r}=0.51, \mathrm{p}=0.001)$. This correlation may further support the use of LVESV index change as an echocardiographic predictor to assess LV remodelling after cardiac resynchronization therapy (CRT).

CONCLUSION: Patients in whom LBBB was diagnosed according to stricter (Strauss's) ECG criteria showed significantly higher intraventricular dyssynchrony and tendency for higher LV wasted work than patients with LBBB according to conventional ECG criteria. Further studies are needed to assess if the same occurs in patients with LVEF $<35 \%$ considered for CRT and further stratification of patients according to ECG pattern may improve the selection of patients who would benefit from CRT. 
Key words: Left bundle branch block, Strauss, Dys-synchrony, CRT, Echocardiography, Left ventricular mechanics.

\section{Introduction:}

Left bundle branch block (LBBB) is an interruption in the normal electrical sequence of activation of the heart muscle. This is reflected by an abnormal pattern seen on the surface electrocardiogram (ECG). This block may occur along the left bundle branch arising from the His-Purkinje system and may result in various ECG patterns (Siegman-Igra et al., 1978). LBBB may be rarely seen in asymptomatic young individuals with a structurally normal heart (Lamb et al., 1960; Imanishi et al., 2006), but it is often associated with underlying heart disease especially when it is of recent onset. These adverse effects of LBBB may be due to the altered sequence of the electrical left ventricular (LV) activation which is then followed by abnormal sequence of LV mechanical contraction even in the absence of structural heart disease. Investigation of the electrical activation pattern in LBBB patients is limited by the presence of comorbidities and the lack of knowledge regarding the duration and extent of the lesion (or lesions).

Identifying complete LBBB on the ECG is not fully standardized and straightforward. Conventional criteria for $\mathrm{LBBB}$, which are widely used clinically and were applied in large trials. In 2009 American Heart Association, American College of Cardiology Foundation and Heart Rhythm Society suggested updates to the conventional diagnostic criteria of LBBB which additionally included broad notched or slurred $\mathrm{R}$ wave in leads I, aVL, V5 and V6 (Surawicz et al., 2009). Strauss et al. in 2011 presented new criteria for diagnosing LBBB in which they emphasized that mid-QRS notching is the key feature of LBBB because these notches denote endocardium and lateral LV wall breakthrough of the depolarization wave front (Strauss et al., 2011).

The abnormal and dyssynchronous contraction in patients with LBBB increases the LV work as while some segments contract, they will stretch the not yet contracting segments, thus resulting in energy loss, waste of myocardial work and ineffective LV ejection (Russell et al., 
2013). LV work could be quantified by using a recently developed and validated noninvasive tool namely regional LV pressure - strain loop area (Russell et al., 2012). Along with segmental and global values for myocardial work, a set of additional indices are also calculated (Russell et al., 2012):

1- Constructive work: it is the positive work performed by a segment during shortening in systole in addition to the negative work during lengthening in isovolumetric relaxation (IVR).

2- Wasted work: it is the negative work performed by a segment during lengthening in systole in addition to the positive work performed during shortening in IVR.

3- Myocardial work efficiency: is estimated by dividing the constructive work by the sum of constructive and wasted work (0-100\%).

Measurement of regional myocardial electrical-mechanical events using velocity data acquired with tissue Doppler imaging (TDI) showed limitation in assessment of dyssynchrony in patient going to have CRT. To overcome these limitations, new parameters were proposed like, the strain delay index (SDI), strain rate dispersion index (SRDI) and mechanical dispersion (MD).

\section{Methods}

\section{Study population:}

We studied 49 asymptomatic subjects with incidental LBBB: 16 with conventional (mean age 69 years, 56\% were men) and 33 with Strauss's criteria (mean age 66 years, 61\% were men). Thorough clinical history and examination of all subjects were done. Standard 12 leads ECG was done and the subjects classified according to the following criteria as in the following table (1) (Surawicz et al., 2009; Strauss et al., 2011): 
Table (1): ECG criteria for diagnosis complete LBBB in adults:

\begin{tabular}{|l|r|r|}
\hline Criteria & $\begin{array}{r}\text { AHA/ACC/HRS } \\
\text { (conventional) }\end{array}$ & D. Strauss \\
\hline QRS duration (m/f, ms) & $\geq 120 / \geq 120$ & $\geq 140 / \geq 130$ \\
\hline $\begin{array}{l}\text { QRS notching or } \\
\text { slurring }\end{array}$ & $\begin{array}{r}\text { Mid-QRS } \\
\text { R wave in leads I, aVL, } \\
\text { V5, and V6 }\end{array}$ & $\begin{array}{r}\text { notching/slurring in } \geq 2 \text { of } \\
\text { leads V1, V2, V5, V6, I, } \\
\text { and aVL }\end{array}$ \\
\hline $\begin{array}{l}\text { QS or rS in leads V1 } \\
\text { and V2 }\end{array}$ & & $\checkmark$ \\
\hline $\begin{array}{l}\text { Delayed intrinsicoid } \\
\text { deflection (>60 ms) }\end{array}$ & $\begin{array}{r}\text { Present in leads V5 and } \\
\text { Vbsent in leads V1, } \\
\text { V2, and V3 }\end{array}$ \\
\hline $\begin{array}{l}\text { Usually discordant ST } \\
\text { and T wave }\end{array}$ & $\checkmark$ & - \\
\hline $\begin{array}{l}\text { Q waves in leads I, V5, } \\
\text { and V6 }\end{array}$ & Absent & $\begin{array}{r}\text { May present in patients } \\
\text { with concomitant anterior } \\
\text { and/or apical infarct }\end{array}$ \\
\hline
\end{tabular}

*ACC: American College of Cardiology

*AHA: American Heart Association

*HRS: Heart Rhythm Society

Then computed tomography coronary angiography (CTCA) was done for all subjects. Those who showed obstructive coronary artery lesions $>50 \%$ were excluded.

Then 2D and 3D echocardiographic assessment of all subjects were done with measurement of:

a. EDV, ESV, LVEF.

b. Global longitudinal strain (GLS).

c. Longitudinal strain delay index (L-SDI).

d. Longitudinal strain rate dispersion index (L-SRDI).

e. Left ventricular mechanical dispersion (LVMD).

f. Septal to posterior wall time to peak radial strain.

g. LV mass, LV sphericity index and LV remodelling index.

h. GWE, GWI, GCW, GWW and WWR. 
Standard transthoracic echocardiography was performed using a Vivid E9 and Vivid E 95 ultrasound system, (GE Vingmed Ultrasound AS, Horten, Norway) equipped with a M5S 3.5- $\mathrm{mHz}$ transducer.

Statistical analysis (Leslie et al., 1991)

Data were expressed as mean \pm standard deviation for continuous variables and as percentages for categorical variables. Between-group comparisons were performed using Student's t-tests or $\mathrm{x} 2$ test, as appropriate. Statistical significance was defined as $p<0.05$. Statistical analysis was performed using SPSS Version 20.0

\section{Results:}

In our study; we studied different echo derived intra-ventricular dys-synchrony indices including classic velocity-time delay indices as well as speckle tracking echo (STE) derived indices and correlating them with myocardial segmental work in subjects with different electrocardiographic morphological patterns of LBBB with no under lying significant cardiac disease except for the electrical abnormality. In another words, "pure LBBB". We recruited prospectively 49 consecutive subjects with $\mathrm{LBBB}$ and applied manual criteria for differentiation of Strauss apart from conventional patterns. Strauss criteria were applied in about $67.3 \%$ versus $32.7 \%$ diagnosed with conventional pattern. The mean age of the all subjects in both groups was $66.82 \pm 14.1$ in our study.

Among dyssynchrony parameters; L-SDI was significantly higher in patients with Strauss's criteria than in conventional one $(37 \pm 17 \%$ vs $25 \pm 10 \%$, respectively, $\mathrm{p}=0.007)$ as seen in table (2). 
Table (2): Comparison between the two studied groups according to L-SDI.

\begin{tabular}{|c|c|c|c|c|c|}
\hline & \multirow{2}{*}{$\begin{array}{c}\text { Total } \\
(n=49)\end{array}$} & \multicolumn{2}{|c|}{ QRS morphology } & \multirow{2}{*}{$\begin{array}{l}\text { Test of } \\
\text { Sig. }\end{array}$} & \multirow[b]{2}{*}{$\mathbf{P}$} \\
\hline & & $\begin{array}{l}\text { Group I } \\
(\mathrm{n}=16)\end{array}$ & $\begin{array}{c}\text { Group II } \\
(\mathbf{n}=\mathbf{3 3})\end{array}$ & & \\
\hline \multicolumn{6}{|l|}{ L-SDI } \\
\hline Min. - Max. & $10.29-81.11$ & $10.29-61.88$ & $14.26-81.11$ & & \\
\hline Mean \pm SD & $33.09 \pm 17.25$ & $24.99 \pm 14.26$ & $37.02 \pm 17.39$ & $\begin{array}{c}\mathrm{U}= \\
138.0^{*}\end{array}$ & $0.007^{*}$ \\
\hline Median & 27.79 & 19.48 & 33.47 & & \\
\hline
\end{tabular}

U: Mann Whitney test

t: Student t-test

$\mathrm{p}$ : $\mathrm{p}$ value for comparing between the studied groups

*: Statistically significant at $\mathrm{p} \leq 0.05$

Group I: $\quad$ LBBB conventional criteria

Group II: $\quad$ LBBB Strauss criteria

Moreover, GWW tended to be higher in patients with Strauss's criteria than in conventional one $(325 \pm 132 \%$ vs $267 \pm 124 \%$ respectively, $\mathrm{p}=0.079$, table 3$)$.

Table (3): Comparison between the two studied groups according to GWW

\begin{tabular}{|c|c|c|c|c|c|}
\hline & \multirow{2}{*}{$\begin{array}{c}\text { Total } \\
(n=49)\end{array}$} & \multicolumn{2}{|c|}{ QRS morphology } & \multirow[b]{2}{*}{$\mathbf{T}$} & \multirow[b]{2}{*}{$\mathbf{P}$} \\
\hline & & $\begin{array}{l}\text { Group I } \\
(n=16)\end{array}$ & $\begin{array}{c}\text { Group II } \\
(\mathbf{n}=\mathbf{3 3})\end{array}$ & & \\
\hline \multicolumn{6}{|l|}{ GWW } \\
\hline Min. - Max. & $81.0-751.0$ & $81.0-480.0$ & $132.0-751.0$ & & \\
\hline Mean \pm SD & $306.6 \pm 133.6$ & $267.9 \pm 128.3$ & $325.3 \pm 134.0$ & 1.424 & 0.079 \\
\hline Median & 300.0 & 254.0 & 316.0 & & \\
\hline
\end{tabular}

t: Student t-test

$\mathrm{p}$ : $\mathrm{p}$ value for comparing between the studied groups

Group I: $\quad$ LBBB conventional criteria

Group II: $\quad$ LBBB Strauss criteria

Left ventricular remodelling index (LVRI) and sphericity index (sp. Index) were found to be positively and negatively -respectively- correlated with GWE, GWI and GCW with statistical significance only in conventional group and not in Strauss group. While GWW was not found to be correlated significantly neither with LVRI nor Sp. Index in both groups (table 4). This means that in patients with conventional criteria of LBBB have better constructive based 
global work indices when they have increased LV mass in excess to LV dilatation, probably as an adaptive mechanism, with less spherical -globular- left ventricular morphology.

Table (4): Correlation between global work indices and LV remodelling parameters

\begin{tabular}{|c|c|c|c|c|c|c|}
\hline & \multicolumn{6}{|c|}{ GWE } \\
\hline & \multicolumn{2}{|c|}{$\begin{array}{l}\text { Total sample } \\
\quad(n=49)\end{array}$} & \multicolumn{2}{|c|}{$\begin{array}{l}\text { Group I } \\
(n=16)\end{array}$} & \multicolumn{2}{|c|}{$\begin{array}{c}\text { Group II } \\
(\mathbf{n}=\mathbf{3 3})\end{array}$} \\
\hline & $\mathbf{R}$ & $\mathbf{P}$ & $\mathbf{R}$ & $\mathbf{P}$ & $\mathbf{R}$ & $\mathbf{P}$ \\
\hline LV RI & 0.324 & $0.031^{*}$ & 0.542 & $0.015^{*}$ & 0.205 & 0.523 \\
\hline \multirow[t]{4}{*}{ Sp. Index } & -0.313 & $0.028^{*}$ & -0.617 & $0.011^{*}$ & -0.148 & 0.412 \\
\hline & \multicolumn{6}{|c|}{ GWI } \\
\hline & \multicolumn{2}{|c|}{$\begin{array}{c}\text { Total sample } \\
\quad(n=49)\end{array}$} & \multicolumn{2}{|c|}{$\begin{array}{l}\text { Group I } \\
(\mathrm{n}=16)\end{array}$} & \multicolumn{2}{|c|}{$\begin{array}{c}\text { Group II } \\
(\mathbf{n}=\mathbf{3 3})\end{array}$} \\
\hline & $\mathbf{R}$ & $\mathbf{P}$ & $\mathbf{R}$ & $\mathbf{P}$ & $\mathbf{r}$ & $\mathbf{P}$ \\
\hline LV RI & 0.305 & $0.033^{*}$ & 0.654 & $0.004^{*}$ & 0.070 & 0.651 \\
\hline \multirow[t]{4}{*}{ Sp. Index } & -0.305 & $0.033^{*}$ & -0.690 & $0.003^{*}$ & -0.085 & 0.638 \\
\hline & \multicolumn{6}{|c|}{ GCW } \\
\hline & \multicolumn{2}{|c|}{$\begin{array}{l}\text { Total sample } \\
\quad(n=49)\end{array}$} & \multicolumn{2}{|c|}{$\begin{array}{l}\text { Group I } \\
(n=16)\end{array}$} & \multicolumn{2}{|c|}{$\begin{array}{c}\text { Group II } \\
(\mathbf{n}=\mathbf{3 3})\end{array}$} \\
\hline & $\mathbf{R}$ & $\mathbf{P}$ & $\mathbf{r}$ & $\mathbf{P}$ & $\mathbf{r}$ & $\mathbf{P}$ \\
\hline LV RI & 0.345 & 0.064 & 0.626 & $0.009^{*}$ & 0.142 & 0.723 \\
\hline \multirow[t]{4}{*}{ Sp. Index } & -0.241 & 0.096 & -0.704 & $0.002^{*}$ & 0.005 & 0.976 \\
\hline & \multicolumn{6}{|c|}{ GWW } \\
\hline & \multicolumn{2}{|c|}{$\begin{array}{c}\text { Total sample } \\
\quad(n=49)\end{array}$} & \multicolumn{2}{|c|}{$\begin{array}{l}\text { Group I } \\
(n=16)\end{array}$} & \multicolumn{2}{|c|}{$\begin{array}{c}\text { Group II } \\
(\mathbf{n}=\mathbf{3 3})\end{array}$} \\
\hline & $\mathbf{R}$ & $\mathbf{P}$ & $\mathbf{r}$ & $\mathbf{P}$ & $\mathbf{r}$ & $\mathbf{P}$ \\
\hline LV RI & -0.131 & 0.254 & -0.081 & 0.657 & -0.196 & 0.401 \\
\hline Sp. Index & 0.203 & 0.161 & 0.051 & 0.852 & 0.175 & 0.329 \\
\hline
\end{tabular}

r: Pearson coefficient

*: Statistically significant at $\mathrm{p} \leq 0.05$

Group I: LBBB conventional criteria

Group II: LBBB Strauss criteria 


\section{Discussion:}

Direct assessment of intra-ventricular LV dys-synchrony in LBBB subjects using traditional qualitative parameters namely abnormal segmental motions like septal flash and apical rocking movements in addition to quantitative parameters like septal to posterior wall delay assessed by time to peak radial strain were assessed in both groups. Septal flash assessed visually and confirmed by TDI was found nearly in $80 \%$ of the whole cohort while apical rocking movement was found in about $85 \%$. Septal to posterior wall time to peak radial strain was found to be less than 130 milli-seconds (commonly used as a cut off for intra-ventricular dys-synchrony in literature) in all subjects (Pitzalis et al., 2002). Comparing both groups shows no significant difference between them regarding these parameters except for the apical rocking movement was significantly present more in Strauss group than conventional one. These contradictory results of qualitative and quantitative parameters signifying intraventricular dys-synchrony urged the need for newer dys-synchrony indices to confirm the coupling of electrical and mechanical dys-synchrony.

In our study we used mechanical dispersion, longitudinal strain delay index and longitudinal strain rate dispersion index as a novel quantitative dys-synchrony index. Prolonged mechanical dispersion reflects increased temporal heterogeneity of myocardial contraction and has been reported to be useful to stratify arrhythmic risk among patients with different cardiac condition (Haugaa et al., 2012; Haugaa et al., 2013; Haland et al., 2016). The mean MD in our study compared to the mean reference value for the same age group as our study population as proposed by Hugo and his colleagues was significantly higher in the whole cohort and consistently higher in both groups as well. The mean in our study was 52.41 $\pm 14.80 \mathrm{msec}$ while the reference physiological value is $41 \pm 10 \mathrm{msec}$ (Rodríguez-Zanella et al., 2017). Despite our study did not prove significant difference between conventional and Strauss groups regarding mechanical dispersion; MD was statistically significant negatively 
correlated with GWE in conventional group and even with higher significance negatively correlated with GWE in Strauss group. It has had also very significant positive correlation with GWW in both groups. These results render MD - as a dys-synchrony parameter- a good predictor of wasted energy in different subsets of LBBB patients and this of importance considering the results of several studies showing a good correlation between CRT responders and post implantation reduction of MD (Van Der Bijl et al., 2017).

Assessment of longitudinal strain delay index (L-SDI) allowed not only to confirm the dys-synchrony pattern seen in LBBB with delayed post systolic shortening of the lateral segments, but also to quantify the potential wasted energy exerted by these segments contracting while other segments are relaxing. It is worthy to be noted that in our study we measured the strain delay index in both delayed post systolic shortening lateral segments as well as early pre-systolic peaking septal segments as they are both lose their effective contribution to ejection phase. Other studies mainly using L-SDI in patients with cardiomyopathy before CRT implantation; used to mark L-SDI in dys-synchronous segments with positive peaks (stretch) more than their negative peaks (contraction) as zero denoting that they are scarred with no significant contractile reserve (Iwano et al., 2011). We adopted the same concept. However; considering our study group population with isolated LBBB and preserved systolic function we did not encounter such a case. In our study; L-SDI was statistically significant higher is Strauss group than conventional one. Also, it had consistent significant negative correlations with GWE, GWI, GCW and significant positive correlation with GWW rendering L-SDI -as a dys-synchrony parameter- a perfect proof of evidence that Strauss LBBB pattern had lower constructive work and higher wasted work mainly due to its higher dys-synchrony pattern more than conventional LBBB pattern.

The concept of the SRDI is based on the following hypothesis. If LV contraction was synchronized, global LV systolic function will theoretically be equal to the average of 
segmental systolic function. Thus, the average of the segmental peak systolic SR, one part of the SRDI, represents the global LV systolic function when contraction is synchronized, whereas global peak systolic SR, the other part of the index, represents that in the presence of dys-synchrony. Therefore, the SRDI, which is the difference between the averaged 18 segments systolic SR and the global LV systolic SR is a direct measure of the magnitude of global dys-synchrony (Marwick, 2006). However; in our study, L-SRDI failed to show significant difference between both groups and failed to show significant correlation with global work analysis parameters.

Direct comparison of both groups regarding global work parameters showed no significant difference regarding GWE, GWI and GCW. This sounded logic considering preselection of the whole cohort with preserved ejection fraction and more importantly healthy subjects with no overt, sub clinical heart failure nor associated obstructive coronary artery disease. However; Strauss group tended to have more wasted work than conventional one (P value $=0.079)$. Indexing the negative work to the positive work to get global wasted work ratio (GWWR) discriminated both groups significantly. Strauss group had significant more GWWR than conventional one $(\mathrm{P}$ value $=0.036)$.

\section{Study limitations}

One limitation that may influence the strain estimation is the artifacts caused by position shift between breath-holds. This complicates the extraction of the epi- and endocardial surfaces, which are needed for defining the region of interest. All speckle-tracking-derived measurements require more capability in image acquisition and, to obtain correct endocardial border delineation, are contingent on the presence of adequate echocardiographic views. The lack of reference values for remodelling indices, strain parameters (except for GLS), global and segmental work indices makes the use of them a matter of relativity with no absolute cut off values. This needs further large-scale studies to evaluate the physiological determinants of 
these parameters in healthy volunteers. Other important limitations of our clinical study include the limited sample size. Further studies including larger sample size applying these parameters and selection criteria on patients with reduced EF candidate for CRT implantation and following up the response can reveal the clinical utility of basic science addressed in our study.

\section{Conclusion:}

In our study; we found that regardless baseline patient demographic and clinical characteristic; electrocardiographic Strauss LBBB pattern with basic echo study shows large LV volumes, spherical configuration and apical rocking movement denotes more dyssynchrony related wasted myocardial work and energy which can be good target for therapy by means of resynchronization therapy with predictable good response. Conventional LBBB pattern may need further dys-synchrony assessment namely higher mechanical dispersion which correlates significantly with GWE. Therefore, the use of novel echo derived dyssynchrony parameters and myocardial work analysis may further characterize and quantify the magnitude of dys-synchrony and help is selecting candidates for re-synchronization therapy with improved outcome and reducing non responders.

\section{References:}

- Haland, T. F., Almaas, V. M., Hasselberg, N. E., Saberniak, J., Leren, I. S., Hopp, E. . . \& Haugaa, K. H. (2016). Strain echocardiography is related to fibrosis and ventricular arrhythmias in hypertrophic cardiomyopathy. European heart journal cardiovascular Imaging, 17(6), 613-621.

- Haugaa, K. H., Goebel, B., Dahlslett, T., Meyer, K., Jung, C., Lauten, A. . . . \& Edvardsen, T. (2012). Risk assessment of ventricular arrhythmias in patients with nonischemic dilated cardiomyopathy by strain echocardiography. Journal of the American Society of Echocardiography, 25(6), 667-673. 
- Haugaa, K. H., Grenne, B. L., Eek, C. H., Ersboll, M., Valeur, N., Svendsen, J. H. . . \& Edvardsen, T. (2013). Strain echocardiography improves risk prediction of ventricular arrhythmias after myocardial infarction. Journal of the American College of Cardiology cardiovascular imaging, 6(8), 841-850.

- Imanishi, R., Seto, S., Ichimaru, S., Nakashima, E., Yano, K., \& Akahoshi, M. (2006). Prognostic significance of incident complete left bundle branch block observed over a 40-year period. The American journal of cardiology, 98(5), 644-648.

- Iwano, H., Yamada, S., Watanabe, M., Mitsuyama, H., Nishino, H., Yokoyama, S. . . \& Tsutsui, H. (2011). Novel strain rate index of contractility loss caused by mechanical dyssynchrony. - A predictor of response to cardiac resynchronization therapy. Circulation journal, 75(9), 2167-2175.

- Lamb, L. E., Kable, K. D., \& Averill, K. H. (1960). Electrocardiographic findings in 67,375 asymptomatic subjects. V. Left bundle branch block. The American journal of cardiology, 6, 130-142.

- Leslie, E., Geoffrey, J., \& James, M. (1991). Statistical analysis. In E. Leslie, J. Geoffrey \& M. James (Eds.), Interpretation and uses of medical statistics ( $^{\text {th }}$ ed p.p. 411-416). USA: Oxford Scientific Publications.

- Marwick, T. H. (2006). Measurement of strain and strain rate by echocardiography: ready for prime time? Journal of the American College of Cardiology, 47(7), 13131327.

- Pitzalis, M. V., Iacoviello, M., Romito, R., Massari, F., Rizzon, B., Luzzi, G. . . . \& Rizzon, P. (2002). Cardiac resynchronization therapy tailored by echocardiographic evaluation of ventricular asynchrony. Journal of the American College of Cardiology, 40(9), 1615-1622.

- Rodríguez-Zanella, H., Boccalini, F., Secco, E., Muraru, D., Aruta, P., Tenaglia, R. . . \& Badano, L. (2017). Left ventricular mechanical dispersion measured with twodimensional speckle tracking echocardiography predicts severe arrhythmic events in patients with ischemic and non-ischemic cardiomyopathy. European heart journal cardiovascular Imaging, 18(Suppl 3), iii80-iii81.

- Russell, K., Eriksen, M., Aaberge, L., Wilhelmsen, N., Skulstad, H., Gjesdal, O. . . \& Smiseth, O. A. (2013). Assessment of wasted myocardial work: a novel method to 
quantify energy loss due to uncoordinated left ventricular contractions. American journal of physiology. Heart and circulatory physiology, 305(7), H996-1003.

- Russell, K., Eriksen, M., Aaberge, L., Wilhelmsen, N., Skulstad, H., Remme, E. W. . . \& Smiseth, O. A. (2012). A novel clinical method for quantification of regional left ventricular pressure-strain loop area: a non-invasive index of myocardial work. European heart journal, 33(6), 724-733.

- Siegman-Igra, Y., Yahini, J. H., Goldbourt, U., \& Neufeld, H. N. (1978). Intraventricular conduction disturbances: a review of prevalence, etiology, and progression for ten years within a stable population of Israeli adult males. American heart journal, 96(5), 669-679.

- Strauss, D. G., Selvester, R. H., \& Wagner, G. S. (2011). Defining left bundle branch block in the era of cardiac resynchronization therapy. The American journal of cardiology, 107(6), 927-934.

- Surawicz, B., Childers, R., Deal, B. J., Gettes, L. S., Bailey, J. J., Gorgels, A. . . \& \& Wellens, H. (2009). AHA/ACCF/HRS recommendations for the standardization and interpretation of the electrocardiogram: part III: intraventricular conduction disturbances: a scientific statement from the American Heart Association Electrocardiography and Arrhythmias Committee, Council on Clinical Cardiology; the American College of Cardiology Foundation; and the Heart Rhythm Society. Endorsed by the International Society for Computerized Electrocardiology. Journal of the American College of Cardiology, 53(11), 976-981.

- Van Der Bijl, P., Khidir, M., Leung, M., Ajmone Marsan, N., Delgado, V., \& Bax, J. J. (2017). Left ventricular mechanical dispersion after cardiac resynchronisation therapy: prognostic implications. European heart journal, 38(Suppl 1), ehx501. 